\title{
Seletividade de Herbicidas Pós-Emergentes na Cultura da MANDIOCA $^{1}$
}

\author{
Selectivity of Post-Emergent Herbicides for Cassava Crop \\ SILVA, D.V. ${ }^{2}$, SANTOS, J.B. ${ }^{3}$, CARVALHO, F.P. ${ }^{2}$, FERREIRA, E.A. ${ }^{4}$, FRANÇA, A.C. ${ }^{2}$, FERNANDES, \\ J.S.C. ${ }^{3}$, GANDINI, E.M.M. ${ }^{5}$ e CUNHA, V.C. ${ }^{5}$
}

\begin{abstract}
RESUMO - A resposta da mandioca à aplicação de herbicidas varia desde a total seletividade até o completo comprometimento da produção devido à intoxicação provocada. Desse modo, objetivou-se neste trabalho avaliar a seletividade de herbicidas aplicados em pós-emergência na mandioca. Para isso, foi conduzido experimento em casa de vegetação, em blocos casualizados com 23 tratamentos (22 herbicidas + testemunha), em quatro repetições. Os herbicidas foram aplicados 60 dias após a brotação da mandioca, quando as plantas apresentavam cerca de 15 folhas completamente expandidas. Avaliou-se semanalmente a toxicidade das plantas e, aos 35 dias após a aplicação, elas foram coletadas para determinação da matéria seca. Os sintomas mais visiveis de intoxicação da mandioca ocorreram 21 dias depois da aplicação, para a maioria dos produtos testados. Ao final do período de avaliação, a mandioca apresentava sinais de recuperação dos danos visuais provocados pelos herbicidas tóxicos à cultura. Ametryn, ametryn + trifloxysulfuron-sodium, atrazine, diuron + hexazinone e sulfentrazone provocaram as maiores reduções de matéria seca e causaram os maiores danos visiveis; de modo contrário, bentazon, fluazifop-p-butil, mesotrione e tembotrione foram os menos tóxicos à cultura. Constataram-se diferentes niveis de seletividade dos herbicidas à cultura, sendo bentazon, fluazifop-p-butil, mesotrione e tembotrione os herbicidas considerados seletivos para uso em programas de manejo de plantas daninhas.
\end{abstract}

Palavras-chave: Manihot esculenta, intoxicação visual, controle químico.

\begin{abstract}
The cassava response to herbicide application varies from complete selectivity to full production loss due to the poisoning caused. Thus, the objective of this work was to evaluate the selectivity of herbicides applied at post-emergence in cassava. An experiment was arranged in a randomized block design, under greenhouse conditions, including 23 treatments $(22$ herbicides + control) and four replications. The herbicides were applied 60 days after cassava shooting, when the plants displayed 15 completely-expanded leaves. Intoxication of cassava plants was evaluated weekly and 35 days after the plants were harvested for dry matter determination. The best visible symptoms of cassava intoxication occurred 21 days after planting for most tested products. At the end of the evaluation period, the cassava plants presented visual signals of recovery from the damage caused by the herbicides. Ametryn, ametryn + trifloxysulfuron-sodium, atrazine, diuron + hexazinone andsulfentrazone caused the greatest reductions of dry matter and the worst visible damage. Contrarily, bentazon, fluazifop-p-butil, mesotrione, and tembotrione were the least toxic to the culture. Cassava presents different levels of selectivity to the herbicides, and bentazon, fluazifopp-butyl, mesotrione, and tembotrione present a potential active principle for use in weed control management programs.
\end{abstract}

Keywords: Manihot esculenta, visual intoxication, chemical control.

1 Recebido para publicação em 15.9.2011 e aprovado em 7.3.2012.

2 Engo-Agro-., M.Sc., Doutorando, Dep. de Fitotecnia, Universidade Federal de Viçosa - DFT/UFV, 36570-000 Viçosa-MG, <danielvaladaos@yahoo.com.br>, <felipepaolinelli@yahoo.com.br>; ${ }^{3}$ Professor Adjunto, Dep. de Agronomia, UFVJM, <barbosa@pq.cnpq.br>,<cabralfranca@yahoo.com.br>, <cunha.fernandes@ufvjm.edu.br>; ${ }^{4}$ Engo-Agroo., D.Sc., Bolsista PNPD, UFVJM, Diamantina-MG, <evanderalves@yahoo.com.br>; ${ }^{5}$ Engenheira-Florestal, Mestre em Produção Vegetal, UFVJM, Diamantina-MG, <elizzandragandini@yahoo.com.br>, <vicunhax@yahoo.com.br>.

Planta Daninha, Viçosa-MG, v. 30, n. 4, p. 835-841, 2012 


\section{INTRODUÇÃO}

A mandioca (Manihot esculenta) é uma planta cultivada em todo o território nacional devido à sua adaptação às mais variadas condições de clima e solo (Viana et al., 2001), sendo considerada importante fonte de alimento humano e animal (Schons et al., 2009).

O Brasil é o segundo maior produtor mundial de mandioca, com produção estimada para o ano de 2011 de 27,1 milhões de toneladas, ocupando uma área de 1,9 milhão de hectares (IBGE, 2011); embora algumas regiões superem a produtividade de $100 \mathrm{t} \mathrm{ha}^{-1}$ de raízes (IITA, 2005), a média nacional é de $14,2 \mathrm{t} \mathrm{ha}^{-1}$ (IBGE, 2011). O fator que mais tem contribuído para esse baixo valor é o manejo inadequado das plantas daninhas, cujo período crítico de controle é de 30 a 120 dias após o plantio (Silva et al., 2012).

Cultivada como anual ou bianual, a mandioca está sujeita à ocorrência de vários ciclos de plantas daninhas, sendo necessária mais de uma intervenção para o controle. Para Azevedo et al. (2000), o problema é agravado pelo fato de a mandioca possuir crescimento inicial lento, deixando o solo descoberto, o que facilita o estabelecimento das plantas daninhas. A presença das plantas daninhas altera o crescimento e o desenvolvimento da cultura, bem como suas características fotossintéticas (Aspiazu et al., 2010), com consequente redução do tamanho, peso e número de raízes.

O método químico é o mais utilizado para controle das plantas daninhas (Silva et al., 2009). Existem no mercado brasileiro 12 herbicidas registrados para a cultura, sendo cinco de princípios ativos alocados em três mecanismos de ação (inibidores do fotossistema II, sintese de carotenoides e da acetil coenzima A carboxilase). Os produtos à base de ametryn, clomazone, isoxaflutole e metribuzin são indicados para aplicação em pré-emergência, e somente o cletodin para pós-emergência, especificamente no manejo das espécies de folhas estreitas (Brasil, 2011).

Silva et al. (2009) e Biffe et al. (2010) ressaltaram a restrita disponibilidade de herbicidas registrados para a cultura, sobretudo para aplicação em pós-emergência da mandioca. De acordo com Oliveira Jr. et al. (1994), a resposta da mandioca à aplicação de herbicidas varia desde a total seletividade para alguns produtos até o completo comprometimento da produção devido à intoxicação provocada.

Diante da busca por novos produtos que possam ser utilizados para controle de plantas daninhas na cultura da mandioca, objetivou-se neste trabalho avaliar a tolerância da mandioca aos herbicidas pós-emergentes aplicados em fase inicial de crescimento.

\section{MATERIAL E MÉTODOS}

O experimento foi conduzido em ambiente protegido, com condições controladas de temperatura e umidade. Utilizou-se de Latossolo Vermelho-Amarelo Distrófico típico, textura argilosa (56\% de argila, $6 \%$ de silte e $38 \%$ de areia). A análise química do solo apresentou pH (água) de 5,4; teor de matéria orgânica de 1,8 daq kg $^{-1} ; \mathrm{P}, \mathrm{K}, \mathrm{Ca}, \mathrm{Mg}, \mathrm{Al}, \mathrm{H}+\mathrm{Al}$ e $\mathrm{CTC}_{\text {efetiva }}$ de 1,$4 ; 10 ; 0,5 ; 0,2 ; 0,4 ; 4,4 ;$ e 1,7 cmolc dm$^{-3}$, respectivamente. Para adequação do substrato quanto à nutrição, foram aplicados o equivalente a $300 \mathrm{~kg} \mathrm{ha}^{-1}$ de calcário dolomítico, $220 \mathrm{~kg} \mathrm{ha}^{-1}$ de superfosfato simples e $40 \mathrm{~kg} \mathrm{ha}^{-1}$ de cloreto de potássio. A adubação nitrogenada foi realizada em cobertura aos 30 dias após a emergência da cultura, na dose de $40 \mathrm{~kg} \mathrm{ha}^{-1}$ de ureia previamente dissolvida em água. As irrigações foram feitas diariamente, por sistema automático de microaspersão.

Os tratamentos constituíram-se de 22 herbicidas de diferentes mecanismos de ação e uma testemunha com ausência de aplicação. Os produtos comerciais e as doses utilizadas estão descritos na Tabela 1. O delineamento experimental utilizado foi o de blocos casualizados com quatro repetições. Cada vaso com capacidade volumétrica de $5 \mathrm{~L}$, contendo solo, representou uma unidade experimental.

As manivas do cultivar IAC-12 foram adquiridas de produtores de mandioca; na sequência, efetuou-se o plantio nos vasos no mês de março de 2010, sendo a brotação visivel cinco dias depois. A aplicação dos herbicidas foi realizada aos 60 dias após o plantio, quando as plantas apresentavam cerca de $20 \mathrm{~cm}$ de 
Tabela 1 - Classificação de herbicidas e respectivas dosagens aplicadas em plantas de mandioca

\begin{tabular}{|c|c|c|c|}
\hline Mecanismo de ação & $\begin{array}{l}\text { Produto } \\
\text { Comercial }\end{array}$ & Nome comum (i.a) & $\begin{array}{c}\text { Dose i.a.* } \\
\left(\mathrm{g} \mathrm{ha}^{-1}\right)\end{array}$ \\
\hline \multirow{8}{*}{ Inibidores do Fotossistema II } & Metrimex & ametryn & 2.500 \\
\hline & Krismat & ametryn + trifloxysulfuron-sodium & $1.280+32,4$ \\
\hline & Primoleo & atrazine & 2.000 \\
\hline & Basagran 600 & bentazon & 720 \\
\hline & Diuron Nortox & diuron & 2.500 \\
\hline & Afalon & linuron & 1.350 \\
\hline & Sencor & metribuzin & 480 \\
\hline & Velpar K & diuron + hexazinone & $1.170+330$ \\
\hline \multirow{4}{*}{ Inibidores da Protoporfirinogênio Oxidase (PPO) } & Flex & fomesafen & 250 \\
\hline & Naja & lactofen & 144 \\
\hline & Goal & oxyfluorfen & 720 \\
\hline & Solara & sulfentrazone & 600 \\
\hline Inibidor da Acetilcoenzima-A Carboxilase (ACCase) & Fusilade & fluazifop-p-butil & 250 \\
\hline Inibidor da ACCase e PPO & Robust & fluazifop-p-butil + fomesafen & $200+250$ \\
\hline \multirow{5}{*}{ Inibidores da Acetolactato Sintase (ALS) } & Classic & chlorimuron-ethyl & 15 \\
\hline & Katana & flazasulfuron & 75 \\
\hline & Sanson & nicosulfuron & 60 \\
\hline & Envoke & trifloxysulfuron-sodium & 7,5 \\
\hline & Equip plus & foramsulfuron + iodosulfuron & $36+2,4$ \\
\hline \multirow{3}{*}{ Inibidores da Síntese de Carotenoides } & Provence & isoxaflutole & 115 \\
\hline & Callisto & mesotrione & 144 \\
\hline & Soberan & tembotrione & 110,8 \\
\hline
\end{tabular}

* i.a. - ingrediente ativo.

altura e cerca de 15 folhas completamente expandidas. Utilizou-se pulverizador costal pressurizado a $\mathrm{CO}_{2}$, com pressão constante, equipado com lança contendo uma ponta tipo leque, trabalhando a uma altura de $50 \mathrm{~cm}$ do alvo, com velocidade de $1 \mathrm{~m} \mathrm{~s}^{-1}$ e volume de calda de $200 \mathrm{~L}^{\text {ha }}{ }^{1}$.

Foram feitas avaliações visuais de intoxicação da cultura aos $7,14,21,28$ e 35 dias após a aplicação dos herbicidas (DAA), com uso de uma escala percentual de notas variando entre 0 (zero) e 100 (cem), em que 0 implica ausência de quaisquer injúrias e 100 a morte da planta.

Aos 35 DAA, foram avaliados altura (AL), diâmetro do caule (DC), número de folhas (NF) e área foliar das plantas de mandioca. Além disso, todo o material vegetal foi colhido, separado em folha, caule e raizes, sendo posteriormente seco em estufa com circulação forçada de ar, a $65{ }^{\circ} \mathrm{C}$, até atingir peso constante, para determinação da matéria seca.

Os dados obtidos foram transformados em porcentagem relativa $(\%$ relativa $=($ valor observado no tratamento/valor observado na testemunha)*100) à testemunha e posteriormente submetidos à análise de variância, e as médias, quando significativas, foram agrupadas segundo critério de Scott-Knott a 5\% de probabilidade de erro.

\section{RESULTADOS E DISCUSSÃO}

Na primeira avaliação, aos sete dias após a aplicação (DAA), foram constatados em todos os tratamentos sintomas visuais de intoxicação de até $17,5 \%$ nas plantas, sendo os valores mais elevados encontrados nos herbicidas inibidores da protoporfirinogênio oxidase - PPO. As plantas apresentavam necrose no 
tecido das folhas, porém elas se recuperavam após o surgimento de novas folhas. Resultado semelhante foi relatado por Silva et al. (2011) para cinco cultivares de mandioca submetidos a crescentes doses da mistura entre o fomesafen e fluazifop-p-butil.

Essa manifestação pode explicar a atribuição das maiores notas aos sintomas de intoxicação até os 14 DAA e a posterior diminuição até os 35 DAA, exceto para o sulfentrazone, que manteve valores elevados e crescentes em todas as avaliações (Tabela 2). Os herbicidas nicosulfuron, atrazine, diuron+hexazinone, ametryn isoladoe com trifloxysulfuron-sodium também provocaram elevada intoxicação às plantas de mandioca.

De acordo com trabalhos de Oliveira Jr. (2001) e Biffe et al. (2010), sintomas mais severos provocados pela mistura de ametryn com clomazone foram observados até os 45 DAA; a partir dessa data, os sintomas de injúrias não foram mais visiveis. O ametryn, apesar de recomendado para aplicação em préemergênciada mandioca, no presente trabalho causou elevada intoxicação quando atingiu as folhas da cultura em fase inicial de crescimento.

Os herbicidas bentazon, fluazifop-p-butil, chlorimuron-ethyl, trifloxysulfuron-sodium, mesotrione e tembotrione apresentaram sintomas de intoxicação inferiores a $22 \%$ nas plantas (Tabela 2). Por sua vez, os herbicidas inibidores da sintese de carotenoides proporcionaram os menores indices de intoxicação, com maior destaque para mesotrione e tembotrione. Silveira et al. (2012) relataram valores de intoxicação pelo mesotrione semelhantes ao observado neste trabalho, confirmando a tolerância da mandioca a esse herbicida.

Observou-se também diferença em altura de plantas, número de folhas e área foliar das plantas de mandioca para os diferentes herbicidas aplicados (Tabela 3). Apenas bentazon, fluazifop-p-butil, mesotrione e tembotrione não

Tabela 2 - Médias de fitotoxicidade em plantas de mandioca aos 7, 14, 21, 28 e 35 dias após a aplicação (DAA) de herbicidas

\begin{tabular}{|c|c|c|c|c|c|c|c|}
\hline \multirow{2}{*}{ Mecanismo de ação } & \multirow{2}{*}{ Tratamento } & \multirow{2}{*}{$\begin{array}{l}\text { Dose i.a } \\
\left(\mathrm{g} \mathrm{ha}^{-1}\right)\end{array}$} & \multicolumn{5}{|c|}{ Média de fitotoxicidade (\%) } \\
\hline & & & $7 \mathrm{DAA}$ & 14 DAA & $21 \mathrm{DAA}$ & $28 \mathrm{DAA}$ & $35 \mathrm{DAA}$ \\
\hline \multirow{8}{*}{ Inibidores do Fotossistema II } & ametryn & 2.500 & $8,75 \mathrm{~b}$ & $35,00 \mathrm{a}$ & $57,50 \mathrm{a}$ & $51,25 \mathrm{a}$ & $67,50 \mathrm{a}$ \\
\hline & ametryn + trifloxysulfuron-sodium & $1.280+32,4$ & $8,75 \mathrm{~b}$ & $20,00 \mathrm{~b}$ & $55,00 \mathrm{a}$ & $63,75 \mathrm{a}$ & $60,00 \mathrm{a}$ \\
\hline & atrazine & 2.000 & $2,50 \mathrm{~d}$ & $15,00 \mathrm{c}$ & $38,75 \mathrm{~b}$ & $45,00 \mathrm{a}$ & $52,50 \mathrm{a}$ \\
\hline & bentazon & 720 & $3,75 \mathrm{c}$ & $8,75 \mathrm{c}$ & $2,50 \mathrm{e}$ & $2,50 \mathrm{c}$ & $6,25 \mathrm{c}$ \\
\hline & diuron & 2.500 & $6,25 \mathrm{c}$ & $15,00 \mathrm{c}$ & $28,75 \mathrm{c}$ & $21,25 \mathrm{~b}$ & $13,75 \mathrm{~b}$ \\
\hline & linuron & 1.350 & $1,25 \mathrm{~d}$ & $8,75 \mathrm{c}$ & $40,00 \mathrm{~b}$ & $20,00 \mathrm{~b}$ & $18,75 \mathrm{~b}$ \\
\hline & metribuzin & 480 & $7,50 \mathrm{~b}$ & $20,00 \mathrm{~b}$ & $36,25 \mathrm{c}$ & $20,00 \mathrm{~b}$ & $22,50 \mathrm{~b}$ \\
\hline & diuron + hexazinone & $1.170+330$ & $5,00 \mathrm{c}$ & $16,25 \mathrm{c}$ & $47,50 \mathrm{~b}$ & $47,50 \mathrm{a}$ & $52,50 \mathrm{a}$ \\
\hline \multirow{4}{*}{ Inibidores da PPO } & fomesafen & 250 & $15,00 \mathrm{a}$ & $40,00 \mathrm{a}$ & $11,25 \mathrm{~d}$ & $11,25 \mathrm{~b}$ & $17,50 \mathrm{~b}$ \\
\hline & lactofen & 144 & $13,75 \mathrm{a}$ & $30,00 \mathrm{a}$ & $23,75 \mathrm{c}$ & $12,50 \mathrm{~b}$ & $12,50 \mathrm{~b}$ \\
\hline & oxyfluorfen & 720 & $15,00 \mathrm{a}$ & $28,75 \mathrm{a}$ & $22,50 \mathrm{c}$ & $20,00 \mathrm{~b}$ & $18,75 \mathrm{~b}$ \\
\hline & sulfentrazone & 600 & $15,00 \mathrm{a}$ & $37,50 \mathrm{a}$ & $28,75 \mathrm{c}$ & $61,25 \mathrm{a}$ & $73,75 \mathrm{a}$ \\
\hline Inibidor da ACCase & fluazifop-p-butil & 250 & $3,75 \mathrm{c}$ & $10,00 \mathrm{c}$ & $5,00 \mathrm{e}$ & $5,00 \mathrm{c}$ & $5,00 \mathrm{c}$ \\
\hline Inibidor da ACCase e PPO & fluazifop-p-butil + fomesafen & $200+250$ & $17,50 \mathrm{c}$ & $41,25 \mathrm{a}$ & $27,50 \mathrm{c}$ & $21,25 \mathrm{~b}$ & $21,25 \mathrm{~b}$ \\
\hline \multirow{5}{*}{ Inibidores da ALS } & chlorimuron-ethyl & 15 & $6,25 \mathrm{c}$ & $6,25 \mathrm{c}$ & $6,25 \mathrm{e}$ & $8,75 \mathrm{c}$ & $8,75 \mathrm{c}$ \\
\hline & flazasulfuron & 75 & $2,50 \mathrm{~d}$ & $8,75 \mathrm{c}$ & $10,00 \mathrm{~d}$ & $15,00 \mathrm{~b}$ & $20,00 \mathrm{~b}$ \\
\hline & nicosulfuron & 60 & $15,00 \mathrm{a}$ & $20,00 \mathrm{~b}$ & $18,75 \mathrm{~d}$ & $26,25 \mathrm{~b}$ & $55,00 \mathrm{a}$ \\
\hline & trifloxysulfuron-sodium & 7,5 & $1,25 \mathrm{~d}$ & $7,50 \mathrm{c}$ & $8,75 \mathrm{e}$ & $2,50 \mathrm{c}$ & $5,00 \mathrm{c}$ \\
\hline & foramsulfuron + iodosulfuron & $36+2,4$ & $5,00 \mathrm{c}$ & $11,25 \mathrm{c}$ & $13,75 \mathrm{~d}$ & $17,50 \mathrm{~b}$ & $27,50 \mathrm{~b}$ \\
\hline \multirow{3}{*}{$\begin{array}{l}\text { Inibidores da Síntese de } \\
\text { Carotenoides }\end{array}$} & isoxaflutole & 115 & $3,75 \mathrm{c}$ & $21,25 \mathrm{~b}$ & $21,25 \mathrm{c}$ & $21,25 \mathrm{~b}$ & $27,50 \mathrm{~b}$ \\
\hline & mesotrione & 144 & $1,25 \mathrm{~d}$ & $7,50 \mathrm{c}$ & $6,25 \mathrm{e}$ & $3,75 \mathrm{c}$ & $2,50 \mathrm{c}$ \\
\hline & tembotrione & 110,8 & $3,75 \mathrm{c}$ & $22,50 \mathrm{~b}$ & $13,75 \mathrm{~d}$ & $11,25 \mathrm{~b}$ & $11,25 \mathrm{c}$ \\
\hline \multicolumn{2}{|c|}{$\mathrm{CV}(\%)$} & - & 22,63 & 20,83 & 17,45 & 19,68 & 19,82 \\
\hline
\end{tabular}

i.a. - ingrediente ativo. Médias seguidas de letras iguais não diferem estatisticamente entre si pelo critério de Scott-Knott a $5 \%$ de significância. 
Tabela 3 - Altura das plantas (AP), número de folhas (NF), área foliar (AF) e diâmetro do caule (DC), em porcentagem em relação à testemunha, de plantas de mandioca aos 35 DAA dos herbicidas

\begin{tabular}{|c|c|c|c|c|c|c|}
\hline Mecanismo de ação & Tratamento & Dose i.a. $\left(\mathrm{g} \mathrm{ha}^{-1}\right)$ & $\mathrm{AP}(\mathrm{cm})$ & $\mathrm{NF}$ & $\mathrm{AF}\left(\mathrm{cm}^{2}\right)$ & $\mathrm{DC}(\mathrm{cm})$ \\
\hline & Testemunha & & $100,00 \mathrm{a}$ & $100,00 \mathrm{a}$ & $100,00 \mathrm{a}$ & $100,00^{\text {ns }}$ \\
\hline \multirow{8}{*}{ Inibidores do Fotossistema II } & ametryn & 2.500 & $78,50 \mathrm{~b}$ & $27,25 \mathrm{c}$ & $24,25 \mathrm{~d}$ & 77,50 \\
\hline & ametryn + trifloxysulfuron-sodium & $1.280+32,4$ & $67,00 \mathrm{c}$ & $44,25 \mathrm{c}$ & $23,50 \mathrm{~d}$ & 77,00 \\
\hline & atrazine & 2.000 & $91,75 \mathrm{a}$ & $44,50 \mathrm{c}$ & $40,25 \mathrm{c}$ & 88,00 \\
\hline & bentazon & 720 & $90,50 \mathrm{a}$ & $93,50 \mathrm{a}$ & $94,25 \mathrm{a}$ & 99,00 \\
\hline & diuron & 2.500 & $91,75 \mathrm{a}$ & $55,75 \mathrm{~b}$ & $80,25 \mathrm{~b}$ & 94,25 \\
\hline & linuron & 1.350 & $79,75 \mathrm{~b}$ & $40,00 \mathrm{c}$ & $52,75 \mathrm{c}$ & 88,25 \\
\hline & metribuzin & 480 & $78,00 \mathrm{~b}$ & $61,25 \mathrm{~b}$ & $56,25 \mathrm{c}$ & 88,75 \\
\hline & diuron + hexazinone & $1.170+330$ & $91,75 \mathrm{a}$ & $37,00 \mathrm{c}$ & $41,75 \mathrm{c}$ & 93,50 \\
\hline \multirow{4}{*}{ Inibidores da PPO } & fomesafen & 250 & $86,25 \mathrm{a}$ & $84,25 \mathrm{a}$ & $69,50 \mathrm{~b}$ & 92,25 \\
\hline & lactofen & 144 & $83,25 \mathrm{~b}$ & $95,75 \mathrm{a}$ & $75,50 \mathrm{~b}$ & 99,00 \\
\hline & oxyfluorfen & 720 & $80,00 \mathrm{~b}$ & $76,50 \mathrm{a}$ & $76,50 \mathrm{~b}$ & 91,75 \\
\hline & sulfentrazone & 600 & $74,50 \mathrm{c}$ & $41,50 \mathrm{c}$ & $19,50 \mathrm{~d}$ & 94,00 \\
\hline Inibidor da ACCase & fluazifop-p-butil & 250 & $79,50 \mathrm{~b}$ & $95,75 \mathrm{a}$ & $86,75 \mathrm{a}$ & 98,00 \\
\hline Inibidor da ACCase e PPO & fluazifop-p-butil + fomesafen & $200+250$ & $83,75 \mathrm{~b}$ & $82,25 \mathrm{a}$ & $72,75 \mathrm{~b}$ & 90,50 \\
\hline \multirow{5}{*}{ Inibidores da ALS } & chlorimuron-ethyl & 15 & $65,00 \mathrm{c}$ & $90,00 \mathrm{a}$ & $58,25 \mathrm{c}$ & 89,75 \\
\hline & flazasulfuron & 75 & $60,25 \mathrm{c}$ & $81,50 \mathrm{a}$ & $50,75 \mathrm{c}$ & 90,50 \\
\hline & nicosulfuron & 60 & $60,50 \mathrm{c}$ & $60,00 \mathrm{~b}$ & $46,00 \mathrm{c}$ & 89,25 \\
\hline & trifloxysulfuron-sodium & 7,5 & $72,00 \mathrm{c}$ & $90,00 \mathrm{a}$ & $70,00 \mathrm{~b}$ & 94,25 \\
\hline & foramsulfuron + iodosulfuron & $36+2,4$ & $74,00 \mathrm{c}$ & $84,25 \mathrm{a}$ & $61,75 \mathrm{c}$ & 83,00 \\
\hline \multirow{3}{*}{$\begin{array}{l}\text { Inibidores da Síntese de } \\
\text { Carotenoides }\end{array}$} & isoxaflutole & 115 & $87,75 \mathrm{~b}$ & $79,25 \mathrm{a}$ & $75,25 \mathrm{~b}$ & 91,00 \\
\hline & mesotrione & 144 & $89,00 \mathrm{a}$ & $95,50 \mathrm{a}$ & $97,50 \mathrm{a}$ & 96,50 \\
\hline & tembotrione & 110,8 & $91,50 \mathrm{a}$ & $95,50 \mathrm{a}$ & $99,50 \mathrm{a}$ & 100,00 \\
\hline \multicolumn{2}{|c|}{ CV $(\%)$} & - & 10,74 & 21,82 & 27,46 & 9,43 \\
\hline
\end{tabular}

i.a. - ingrediente ativo. Médias seguidas de letras iguais não diferem estatisticamente entre si pelo critério de Scott-Knott a 5\% de significância.

diferiram da testemunha nas variáveis avaliadas.

Os herbicidas inibidores da enzima acetolactato sintase (ALS) causaram reduções superiores a 25, 10 e $30 \%$ em altura, número de folhas e área foliar das plantas, respectivamente. A observação deve-se à capacidade desses herbicidas de inibir o crescimento das plantas quase que imediatamente após a aplicação (Vidal \& Winkler, 2002).

As mesmas variáveis foram reduzidas pela ação dos herbicidas ametryn e sulfentrazone e da mistura ametryn+trifloxysulfuron-sodium (Tabela 3). O ametryn é recomendado para a cultura da mandioca em aplicação anterior à brotação, e os resultados encontrados evidenciam a intoxicação provocada pelo herbicida para aplicações sobre as folhas, promovendo redução no crescimento. Uma alternativa seria a aplicação das misturas de ametryn+ clomazone e diuron quando a mandioca estiver com somente com 5 a $10 \%$ das brotações emergidas, conforme sugerido por Oliveira Jr. et al. (2001).

Quanto ao acúmulo de matéria seca de folhas, verificou-se sensibilidade da aplicação dos herbicidas; apenas os tratamentos à base de bentazon, fluazifop-p-butil, mesotrione e tembotrione não diferiram estatisticamente da testemunha (Tabela 4). Para os herbicidas ametryn, ametryn+trifloxysulfuron-sodium, atrazine, diuron+hexazinone e sulfentrazone, as reduções foram maiores que $60 \%$. Essa queda refletiu na desfolha da mandioca provocada por esses herbicidas e consequente redução da área foliar da planta (Tabela 3). De acordo com Viana et al. (2001), a redução do crescimento da parte aérea contribui para diminuição do tecido fotossintético, o que prejudica o acúmulo de carboidratos para as raízes, afetando a produção final da cultura. 
Tabela 4 - Matéria seca total (MST), de raízes (MSR), de folhas (MSF) e do caule (MSC), em porcentagem em relação à testemunha, aos 35 DAA dos herbicidas

\begin{tabular}{|c|c|c|c|c|c|c|}
\hline Mecanismo de ação & Tratamento & Dose i.a. $\left(\mathrm{g} \mathrm{ha}^{-1}\right)$ & MSF & MSC & MSR & MST \\
\hline & Testemunha & - & $100,00 \mathrm{a}$ & $100,00 \mathrm{a}$ & $100,00 \mathrm{a}$ & $100,00 \mathrm{a}$ \\
\hline \multirow{8}{*}{ Inibidores do Fotossistema II } & ametryne & 2.500 & $12,50 \mathrm{~d}$ & $33,25 \mathrm{~b}$ & $45,75 \mathrm{~b}$ & $23,75 \mathrm{~d}$ \\
\hline & ametryne + trifloxysulfuron-sodium & $1.280+32,4$ & $18,25 \mathrm{~d}$ & $36,50 \mathrm{~b}$ & $33,75 \mathrm{~b}$ & $25,00 \mathrm{~d}$ \\
\hline & atrazine & 2.000 & $25,50 \mathrm{~d}$ & $42,00 \mathrm{~b}$ & $36,50 \mathrm{~b}$ & $31,25 \mathrm{~d}$ \\
\hline & bentazon & 720 & $87,75 \mathrm{a}$ & $92,00 \mathrm{a}$ & $91,75 \mathrm{a}$ & $92,75 \mathrm{a}$ \\
\hline & diuron & 2500 & $71,75 \mathrm{~b}$ & $75,00 \mathrm{a}$ & $67,50 \mathrm{a}$ & $74,00 \mathrm{~b}$ \\
\hline & linuron & 1350 & $43,00 \mathrm{c}$ & $57,75 \mathrm{~b}$ & $67,75 \mathrm{a}$ & $56,00 \mathrm{c}$ \\
\hline & metribuzin & 480 & $49,50 \mathrm{c}$ & $65,00 \mathrm{a}$ & $69,00 \mathrm{a}$ & $56,75 \mathrm{c}$ \\
\hline & diuron + hexazinone & $1.170+330$ & $31,50 \mathrm{~d}$ & $60,75 \mathrm{~b}$ & $34,25 \mathrm{~b}$ & $38,00 \mathrm{~d}$ \\
\hline \multirow{4}{*}{ Inibidores da PPO } & fomesafen & 250 & $62,25 \mathrm{~b}$ & $75,25 \mathrm{a}$ & $79,00 \mathrm{a}$ & $74,75 \mathrm{~b}$ \\
\hline & lactofen & 144 & $73,00 \mathrm{~b}$ & $86,75 \mathrm{a}$ & $76,50 \mathrm{a}$ & $78,50 \mathrm{~b}$ \\
\hline & oxyfluorfen & 720 & $73,50 \mathrm{~b}$ & $72,25 \mathrm{a}$ & $67,25 \mathrm{a}$ & $74,75 \mathrm{~b}$ \\
\hline & sulfentrazone & 600 & $20,50 \mathrm{~d}$ & $70,75 \mathrm{a}$ & $79,50 \mathrm{a}$ & $45,50 \mathrm{c}$ \\
\hline Inibidor da ACCase & fluazifop-p-butil & 250 & $87,75 \mathrm{a}$ & $76,25 \mathrm{a}$ & $82,00 \mathrm{a}$ & $87,50 \mathrm{a}$ \\
\hline Inibidor da ACCase e PPO & fluazifop-p-butil + fomesafen & $200+250$ & $70,50 \mathrm{~b}$ & $77,00 \mathrm{a}$ & $82,00 \mathrm{a}$ & $75,75 \mathrm{~b}$ \\
\hline \multirow{5}{*}{ Inibidores da ALS } & \begin{tabular}{|c|} 
chlorimuron-ethyl \\
\end{tabular} & 15 & $55,50 \mathrm{~b}$ & $73,75 \mathrm{a}$ & $64,00 \mathrm{a}$ & $65,25 \mathrm{~b}$ \\
\hline & flazasulfuron & 75 & $54,50 \mathrm{~b}$ & $70,50 \mathrm{a}$ & $53,25 \mathrm{~b}$ & $60,75 \mathrm{c}$ \\
\hline & nicosulfuron & 60 & $52,75 \mathrm{~b}$ & $81,00 \mathrm{a}$ & $60,25 \mathrm{~b}$ & $61,50 \mathrm{c}$ \\
\hline & trifloxysulfuron-sodium & 7,5 & $65,75 \mathrm{~b}$ & $75,25 \mathrm{a}$ & $84,00 \mathrm{a}$ & $73,25 \mathrm{~b}$ \\
\hline & foramsulfuron + iodosulfuron & $36+2,4$ & $57,50 \mathrm{~b}$ & $66,00 \mathrm{a}$ & $82,75 \mathrm{a}$ & $75,50 \mathrm{~b}$ \\
\hline \multirow{3}{*}{$\begin{array}{l}\text { Inibidores da Síntese de } \\
\text { Carotenoides }\end{array}$} & isoxaflutole & 115 & $62,75 \mathrm{~b}$ & $59,75 \mathrm{~b}$ & $50,00 \mathrm{~b}$ & $61,25 \mathrm{~b}$ \\
\hline & mesotrione & 144 & $96 \mathrm{a}$ & $91,75 \mathrm{a}$ & $99 \mathrm{a}$ & $98,25 \mathrm{a}$ \\
\hline & tembotrione & 110,8 & $95 \mathrm{a}$ & $93,75 \mathrm{a}$ & $81,5 \mathrm{a}$ & $93,75 \mathrm{a}$ \\
\hline & $\mathrm{CV}(\%)$ & - & 30,41 & 26,04 & 31,13 & 26,38 \\
\hline
\end{tabular}

i.a. - ingrediente ativo. Médias seguidas de letras iguais não diferem estatisticamente entre si pelo critério de Scott-Knott a 5\% de significância.

A matéria seca do caule foi afetada pela maioria dos produtos aplicados (Tabela 4), particularmente pelos inibidores do fotossistema II. A mandioca propaga-se de forma vegetativa, através das ramas que são retiradas de segmentos das hastes da parte aérea; assim, qualquer impacto no crescimento do caule, em fase inicial de desenvolvimento, pode acarretar redução da qualidade do material de plantio e alterar a produção de safras posteriores.

De acordo com Peressin \& Carvalho (2002), dos 7 aos 90 dias após o plantio da maniva ocorre a fase de formação do sistema radicular, principalmente das raízes fibrosas, algumas das quais irão se transformar em raizes de armazenamento. No que se refere aos efeitos dos herbicidas na matéria seca das raízes, constatou-se que ametryn, ametryn + trifloxysulfuron-sodium, atrazine, diuron + hexazinone e isoxaflutole causaram maiores reduções. Apenas bentazon, fluzifop-p-butil, mesotrione e tembotrione não causaram reduções significativas na matéria seca total das plantas de mandioca. Esses resultados estão de acordo com os apresentados na Tabela 2, sendo esses herbicidas os que causaram os menores indices de intoxicação à cultura.

Os herbicidas bentazon, fluazifop-p-butil, mesotrione e tembotrione mostraram-se mais promissores para aplicação em área total, uma vez que foram altamente seletivos à cultura da mandioca até os 35 DAA. Portanto, esses herbicidas apresentam potencial para utilização em programas de controle de plantas daninhas, constituindo-se em opções de mecanismos de ação distintos.

\section{AGRADECIMENTOS}

À Coordenação de Aperfeiçoamento de Pessoal de Nivel Superior (Capes), ao Conselho Nacional de Desenvolvimento Científico e Tecnológico (CNPq) e à Fundação de Amparo à Pesquisa do Estado de Minas Gerais (FAPEMIG), 
pelo apoio financeiro na execução deste trabalho.

\section{LITERATURA CITADA}

ASPIAZU, I. et al. Photosynthetic activity of cassava plants under weed competition. Planta Daninha, v. 28, p. 963-968, 2010. (Número Especial)

AZEVÊDO, C. L. L. et al. Levantamento de plantas daninhas na cultura da mandioca, em um ecossistema semi-árido do Estado da Bahia. Magistra, v. 12, n. 1/2, 2000. Disponível em: $<$ http://www.magistra.ufba.br/publica/magist $>$. Acesso em: 15 mar. 2011.

BIFFE, D. F. et al. Avaliação de herbicidas para dois cultivares de mandioca. Planta Daninha, v. 28, n. 4, p. 807-816, 2010.

\section{INSTITUTO BRASILEIRO DE GEOGRAFIA E}

ESTATÍSTICA - IBGE. Sétima previsão da safra 2011/2012. Disponível em: < http://www1.ibge.gov.br/home/presidencia/ noticias/noticia_visualiza.php?id_noticia $=1798 \& i d$ pagina $=$ 1>. Acesso em: 10 abr. 2011.

IITA. Cassava productivity in the lowland and midaltitude agroecologies of sub saharan Africa. Disponível em: < http://www.iita.org/cms/articlefiles/92 IITA\%20MTP\%202001_2003.pdf >. Acesso em: 30 mar. 2010.

PERESSIN, V. A.; CARVALHO, J. E. B. Manejo integrado de plantas daninhas em mandioca. In: CEREDA, M. P. (Org.).

Cultura de tuberosas amiláceas latino-americanas. São Paulo: Fundação Cargill, 2002.v. 2. p. 3.

BRASIL. Ministério da Agricultura, Pecuária e Abastecimento. Coordenação-Geral de Agrotóxicos e Afins. Disponível em: $<$.http://extranet.agricultura.gov.br / agrofit_cons/principal_agrofit_cons $>$. Acesso em: 9 abr. 2011 .
OLIVEIRA JR., R. S. et al. Tolerância de cinco cultivares de mandioca (Manihot esculenta) a herbicidas. Planta Daninha, v. 19, n. 1, p. 119-125, 2001.

OLIVEIRA JR., R. S. Seletividade e eficiência de trifluralin e diuron aplicados em diferentes formas na cultura da mandioca (Manihot esculenta Crantz). R. Unimar, v. 16, n. 2, p. 317-325, 1994.

SCHONS, A. et al. Arranjos de plantas de mandioca e milho em cultivo solteiro e consorciado: crescimento, desenvolvimento e produtividade. Bragantia, v. 68 , n. 1, p. 155-167, 2009.

SILVA, F. M. L. et al. Moléculas de herbicidas seletivos à cultura da mandioca. R. Trópica, v. 3, n. 2, p. 61-72, 2009.

SILVA, D.V. et al. Manejo de plantas daninhas na cultura da mandioca. Planta Daninha, v. 30, n. 3, p. ??, 2012. (no prelo).

SILVA, D. V. et al. Tolerância de cultivares de mandioca aos herbicidas fomesafen e fluazifop-p-butil. R. Bras.

Herbicidas, v. 10, n. 3, p. 219-231, 2011.

SILVEIRA, H. M. et al. Sensibilidade de cultivares de mandioca ao herbicida mesotrione. R. Bras. Herbicidas, v. 11, n. 1 , p. $24-31,2011$..

VIANA, A. E. S.; SEDIYAMA, T.; CECON, P. R. Efeito do comprimento e de incisões no córtex da maniva sobre o cultivo da mandioca (Manihot esculenta Crantz). Acta Sci., v. 23, n. 5, p. 1263-1269, 2001.

VIDAL, R. A.; WINKLER, L. M. Resistência de plantas daninhas: seleção ou indução à mutação pelos herbicidas inibidores de acetolactato sintase (ALS). Pestic. R. Ecotoxicol. Meio Amb., v. 12, n. 1, p. 31-42, 2002. 